\title{
Streptococcus pneumoniae coinfection in hospitalised patients with COVID-19
}

\author{
Vanesa Anton-Vazquez ${ }^{1}$ (D) $\cdot$ Raquel Clivillé ${ }^{2}$
}

Received: 8 December 2020 / Accepted: 13 January 2021 / Published online: 19 January 2021

(C) The Author(s), under exclusive licence to Springer-Verlag GmbH, DE part of Springer Nature 2021

To the Editor,

We would like to share our experience of a series of COVID19 patients coinfected with pneumococcal pneumonia in a district hospital in Barcelona. Bacterial coinfections in patients with COVID-19 are uncommon [1] including those caused by Streptococcus pneumoniae [2], especially when compared with other seasonal respiratory viruses [3]. However, the evidence on bacterial pneumonia in COVID19 is still limited and diagnosis remains a challenge, as both diseases are associated with a similar clinical presentation, high inflammatory markers and radiological changes. Given the high mortality associated with S. pneumoniae infection, missing the diagnosis may pose a risk to already vulnerable COVID-19 patients. As such, we describe the prevalence, clinical characteristics and outcomes of pneumococcal infection in COVID-19 patients.

We reviewed all microbiology results for patients admitted to Hospital Sant Joan Despí Moises Broggi (Barcelona, Spain) with PCR-confirmed COVID-19 on nasopharyngeal swabs between March 4, 2020, and November 4, 2020. Our hospital serves a population of 425.000 , of which one-quarter are foreign-born, mainly Latin-American. Hospital guidelines for patients admitted with COVID-19 pneumonia recommend blood culture, pneumococcal and legionella urinary antigen tests based on clinical severity and at the clinician's discretion. Clinical samples were tested for pneumococcal antigen using a fluorescence immunoassay, SD Biosensor S. pneumoniae urinary antigen test (Republic of Korea), in accordance with

Vanesa Anton-Vazquez

vantonva@sgul.ac.uk; Vanesa.anton.v@gmail.com

1 Department of Internal Medicine, Hospital Sant Joan Despí Moisès Broggi, Barcelona, Spain

2 Department of Clinical Microbiology, Consorci del Laboratori Intercomarcal de l'Alt Penedès, l'Anoia i el Garraf (CLILAB Diagnòstics), Hospital Sant Joan Despí Moisès Broggi, Barcelona, Spain the manufacturer's instructions. Two different kits were used to detect SARS-CoV-2 from nasopharyngeal samples: Multiplex PCR, Cepheid, PCR SARS-CoV-2 GeneXpert (USA) and Multiplex PCR, Diasorin, PCR SARS-CoV-2 Simplexa (USA). The treatment of COVID-19 infection was based on hydroxychloroquine and azithromycin from March to June 2020 and dexamethasone with or without remdesivir (according to local protocol) from July to November 2020. Antibiotic treatment used to treat pneumococcal pneumonia was ceftriaxone in the great majority of cases.

Eighty-seven (3\%) patients of a total of 2782 who tested positive for COVID-19 and 9\% of 917 patients specifically tested for pneumococcal antigen had a pneumococcal coinfection. Of those 87 coinfected patients, $50(57 \%)$ were females, mean age was 68 years (range, 27-92), and 22 (25\%) were foreign-born, mainly from Latin-America (17\%). Hypertension $47 \%$, diabetes $33 \%$, obesity $22 \%$, malignancy $15 \%$ and chronic lung disease $11 \%$ were the main comorbidities. Chest X-ray showed bilateral infiltrates in $73(84 \%)$ cases and consolidation was obvious in only 14 (16\%) cases. Thirty-day mortality was $17 \%$ (15/87). COVID-19 and pneumococcal pneumonia coinfected patients compared with COVID-19 individuals with a negative pneumococcal testing were mostly female ( $57 \%$ vs $34 \%, p<0.001)$. No differences in age, comorbidities, ethnicity, length of stay, admission to intensive care unit or 30-day mortality were found between groups. Patient demographics, clinical characteristics and outcomes are shown in Table 1.

Despite the low prevalence of pneumococcal pneumonia in COVID-19 patients (3\%), this was higher than the one reported in other studies that varied from 1.2 to $2.5 \%$ [4] [2]. The higher prevalence of $S$. pneumoniae and SARS-CoV2 coinfection may be due to a sub-optimal pneumococcal vaccination rates in Spain, ranging from $35 \%$ in high-risk population aged $<65$ years to $63 \%$ in older adults $\geq 65$ years [5]. This vaccination coverage rate is below the $90 \%$ target set by public health immunisation goals. In addition, lower pneumococcal vaccination rates have also been found among different 
Table 1 Patient demographics, clinical characteristics and outcomes of COVID-19-infected patients with positive and negative pneumococcal urinary antigen. Descriptive statistics are summarised using frequencies and percentages or medians and interquartile ranges (IQR). $p$ values were calculated using $\chi^{2}$ for categorical and MannWhitney $U$ test for continuous variables using SPSS v.26. $p$ values $<0.05$ were considered significant

\begin{tabular}{|c|c|c|c|c|c|}
\hline \multirow[b]{2}{*}{ Age (years) median (range) } & \multicolumn{2}{|c|}{$\begin{array}{l}\text { Pneumococcal Ag positive, } \\
n=87\end{array}$} & \multicolumn{2}{|c|}{$\begin{array}{l}\text { Pneumococcal Ag } \\
\text { negative, } n=830\end{array}$} & \multirow{2}{*}{$\begin{array}{l}p \text { value } \\
0.090\end{array}$} \\
\hline & 68 & $(27-92)$ & 65 & $(21-97)$ & \\
\hline \multicolumn{6}{|l|}{ Gender $(\%)$} \\
\hline Female & 50 & $(57 \%)$ & 285 & $(34 \%)$ & $<0.001$ \\
\hline \multicolumn{6}{|l|}{ Ethnicity (\%) } \\
\hline Spanish & 65 & $(75 \%)$ & 625 & $(75 \%)$ & 0.897 \\
\hline Foreign-born & 22 & $(25 \%)$ & 205 & $(25 \%)$ & 0.108 \\
\hline Latin-American & 15 & $(17 \%)$ & 178 & $(21 \%)$ & 0.409 \\
\hline Indian/ Pakistan & 5 & $(6 \%)$ & 5 & $(1 \%)$ & 0.001 \\
\hline Moroccans & 3 & $(3 \%)$ & 23 & $(3 \%)$ & 0.730 \\
\hline Comorbidities (\%) & 63 & $(72 \%)$ & 535 & $(65 \%)$ & 0.156 \\
\hline Hypertension & 41 & $(47 \%)$ & 411 & $(50 \%)$ & 0.736 \\
\hline Diabetes mellitus & 29 & $(33 \%)$ & 210 & $(25 \%)$ & 0.123 \\
\hline Obesity & 19 & $(22 \%)$ & 143 & $(17 \%)$ & 0.301 \\
\hline Malignancy & 13 & $(15 \%)$ & 74 & $(9 \%)$ & 0.082 \\
\hline Chronic lung disease & 10 & $(11 \%)$ & 96 & $(12 \%)$ & 0.949 \\
\hline \multicolumn{6}{|l|}{ Laboratory results, median (range) } \\
\hline White cell count $(10 \times 9 / \mathrm{L})$ & 8 & $(1-30)$ & 7 & $(1.3-3.3)$ & 0.982 \\
\hline Lymphocytes $(10 \times 9 / \mathrm{L})$ & 1.1 & $(0.3-3.7)$ & 1.05 & $(0.1-3.1)$ & 0.352 \\
\hline $\mathrm{CRP}(\mathrm{mg} / \mathrm{L})$ & 111 & $(7-434)$ & 87 & $(1-594)$ & 0.396 \\
\hline D-Dimer (ng/mL) & 1020 & $(250-5100)$ & 860 & $(3-20000)$ & 0.279 \\
\hline \multicolumn{6}{|l|}{ Clinical outcomes } \\
\hline Length of stay (days), median (range) & 9 & $(1-35)$ & 9 & $(1-90)$ & 0.982 \\
\hline Intensive care unit admission (\%) & 8 & $(9 \%)$ & 132 & $(16 \%)$ & 0.117 \\
\hline 30-day mortality (\%) & 15 & $(17 \%)$ & 129 & $(16 \%)$ & 0.644 \\
\hline \multicolumn{6}{|l|}{ Radiological findings (chest X ray) (\%) } \\
\hline Bilateral infiltrates & 73 & $(84 \%)$ & 741 & $(89 \%)$ & 0.105 \\
\hline Consolidation & 14 & $(16 \%)$ & 24 & $(3 \%)$ & 0.001 \\
\hline No infiltrates/interstitial changes & 0 & $(0 \%)$ & 62 & $(8 \%)$ & 0.001 \\
\hline
\end{tabular}

ethnic minorities [6], which may have further contributed to higher S. pneumoniae coinfections in our population. Given the nature of this retrospective study, the diagnostic tests for pneumococcal infection were performed and interpreted by the attending physician based on clinical details, and consequently possible false positive results were not taken into consideration. Although pneumococcal urinary antigen specificity has been shown to be as high as 94-97\%, false positive results may occur in patients with recent pneumococcal infection or immunisation [7].

Diagnosis of pneumococcal pneumonia is likely to be missed due to non-specific clinical features and lack of laboratory and radiological specific findings for bacterial pneumonia in patients affected by COVID-19. Therefore, coinfection with $S$. pneumoniae should be suspected in COVID-19 patients and traditional microbiology techniques such as urinary antigen test should be routinely performed. Further understanding of the pathogenesis of pneumococcal pneumonia in
COVID-19 may be useful to better characterise patients at risk of coinfection, particularly in populations with low pneumococcal vaccination rates. Additional studies of COVID-19 patients coinfected with $S$. pneumoniae are needed to further investigate if there exist any gender disparities in susceptibility to bacterial coinfection in COVID-19. Finally, reinforcing pneumococcal vaccination during all healthcare encounters is highly recommended to increase coverage rates and prevent S. pneumoniae disease in COVID-19 patients.

\section{References}

1. Lansbury L, Lim B, Baskaran V, Lim WS (2020) Co-infections in people with COVID-19: a systematic review and meta-analysis. J Infect 81(2):266-275

2. Adler H, Ball R, Fisher M, Mortimer K, Vardhan MS (2020) Low rate of bacterial co-infection in patients with COVID-19. Lancet Microbe 1(2):e62 
3. Metersky ML, Masterton RG, Lode H, File TM Jr, Babinchak T (2012) Epidemiology, microbiology, and treatment considerations for bacterial pneumonia complicating influenza. Int J Infect Dis 16(5):e321-e331

4. Garcia-Vidal C, Sanjuan G, Moreno-García E, Puerta-Alcalde P, Garcia-Pouton N, Chumbita M et al (2020) Incidence of coinfections and superinfections in hospitalized patients with COVID-19: a retrospective cohort study. Clin Microbiol Infect.

5. Vila-Córcoles A, Ochoa-Gondar O, de Diego C, Satué E, VilaRovira A, Aragón M. Pneumococcal vaccination coverages by age, sex and specific underlying risk conditions among middle-aged and older adults in Catalonia, Spain, 2017. Euro Surveill. 2019;24(29).
6. Lu PJ, O'Halloran A, Williams WW, Lindley MC, Farrall S, Bridges CB (2015) Racial and ethnic disparities in vaccination coverage among adult populations in the U.S. Am J Prev Med. 49(6 Suppl 4):S412-S425

7. Sinclair A, Xie X, Teltscher M, Dendukuri N (2013) Systematic review and meta-analysis of a urine-based pneumococcal antigen test for diagnosis of community-acquired pneumonia caused by Streptococcus pneumoniae. J Clin Microbiol. 51(7):2303-2310

Publisher's note Springer Nature remains neutral with regard to jurisdictional claims in published maps and institutional affiliations. 